\title{
Magnetic Phase Imaging Using Interferometric STEM
}

Alice Greenberg, Benjamin McMorran, Cameron Johnson and Fehmi Yasin

University of Oregon, Eugene, Oregon, United States

Several TEM imaging modes can be employed to measure nanoscale magnetic fields. Off-axis electron holography can measure magnetic phase shifts relative to a vacuum reference, and Lorentz TEM and differential phase contrast (DPC) microscopy measure gradients in magnetic phase. All of these datasets can be processed to reconstruct the in-plane vector components of the projected magnetic field. Each of these techniques has advantages and disadvantages to each other. For example, magnetic phase contrast in DPC and Lorentz TEM is limited by the magnitude of the phase gradients produced in the sample, and off-axis holography requires highly coherent sources as well as an additional active element in the system - a biprisms.

We have been developing a separated-path STEM interferometry technique [1-4] and have begun to apply it to image nanoscale magnetic texture [5,6]. This arrangement introduces an amplitude-dividing beam splitter in the probe-forming STEM optics of the instrument, such that each probe electron traverses a superposition of two paths. One path forms a probe beam and the other forms a reference beam traversing vacuum. The relative phase between these two beams is recorded by taking an image of the far-field interference pattern using a direct electron imaging detector. Thus, this an interferometric 4D-STEM technique in which the phase of the bright field disc is directly measured.

We present our latest application of this technique to the study of magnetic fields. We examine the magnetic-induced phase within patterned squares of permalloy thin film, and experimentally observe the expected pyramidal phase structure associated with the magnetic flux closure domain (Fig. 1). We also perform 2-path interferometric STEM on FeGd thin films in which we observe magnetic skyrmions using Lorentz TEM [7-9]. Interferometric phase images near an edge of the FeGd thin film reveal bubble-like features (Fig. 2a). The gradient of this phase image returns a reconstructed magnetic induction map (Fig. 2b) that features expected magnetic skyrmions (Fig. 2c) that we also observed with Lorentz TEM. We will discuss the challenges of this technique and ways to improve it. These results represent a further development of interferometric STEM as applied to magnetic imaging [10]. 


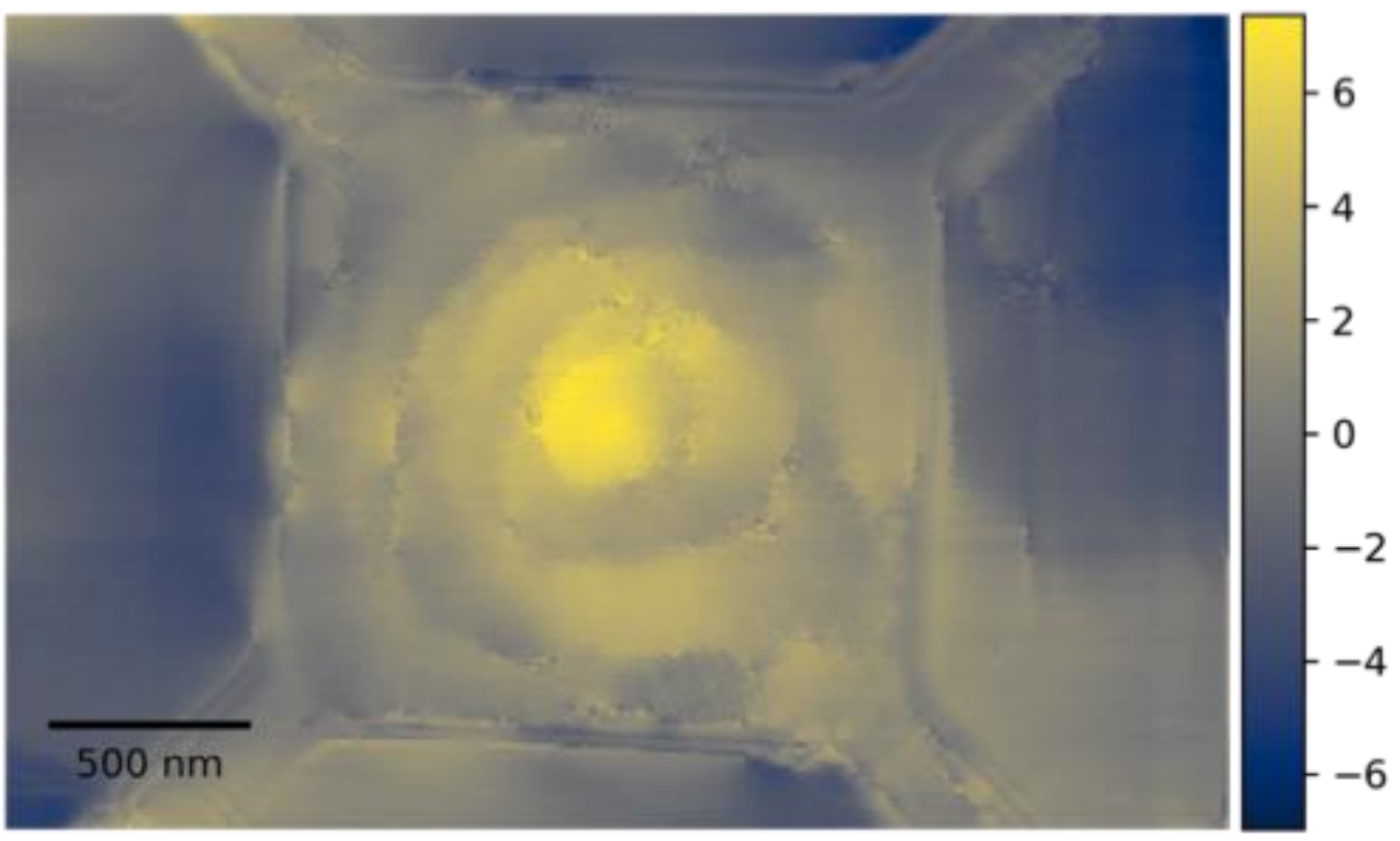

Figure 1. Interferometric STEM phase image of a Landau magnetic domain in a suspended square of permalloy thin film.

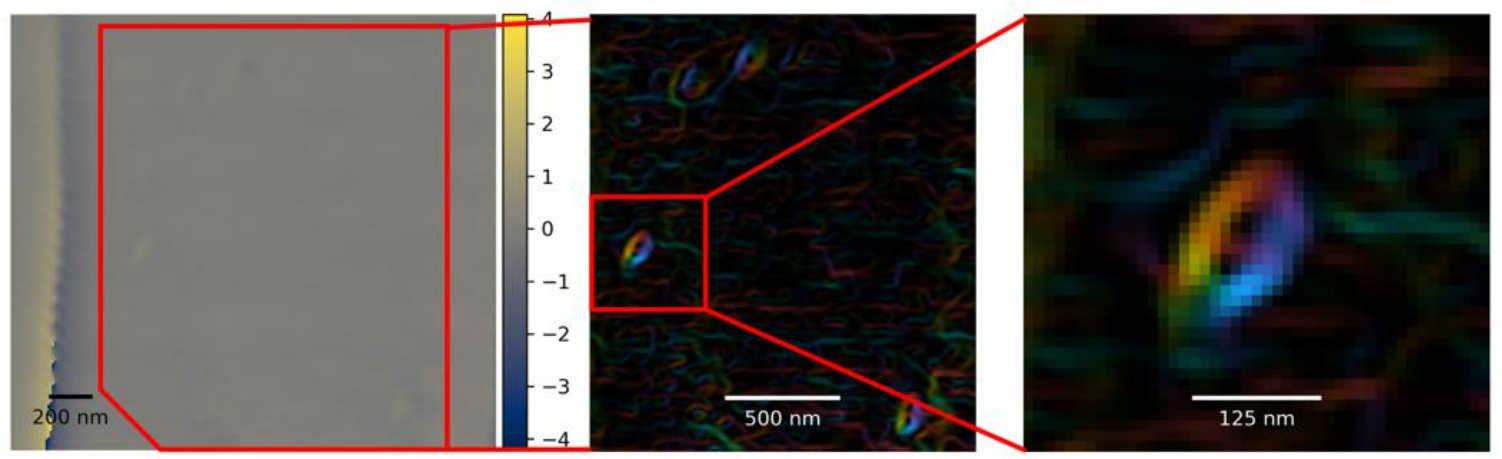

Figure 2. Interferometric STEM image of magnetic features in FeGd thin film. (Left) Phase image mapped by measuring the phase shift of the sample probe relative to the vacuum reference probe at each probe position. The edge of the thin film can be seen on the left and slight "bubbles" in the phase are indicative of magnetic skyrmions. (Middle) The magnetic induction map calculated from the gradient of the phase image reveals magnetic features. (Right) Raw images of magnetic bubbles likely correspond to magnetic skyrmions or pairs of skyrmions called bi-skyrmions, which we have observed in these films using Lorentz TEM.

\section{References}

1. F. S. Yasin, T. R. Harvey, J. J. Chess, J. S. Pierce, and B. J. McMorran, "Development of STEMHolography," Microsc. Microanal. 22, 506-507 (2016).

2. F. S. Yasin, T. R. Harvey, J. J. Chess, J. S. Pierce, and B. J. McMorran, "Path-separated electron interferometry in a scanning transmission electron microscope," J. Phys. Appl. Phys. 51, 205104 (2018). 3. T. R. Harvey, F. S. Yasin, J. J. Chess, J. S. Pierce, R. M. S. dos Reis, V. B. Özdöl, P. Ercius, J. Ciston, W. Feng, N. A. Kotov, B. J. McMorran, and C. Ophus, "Interpretable and Efficient Interferometric 
Contrast in Scanning Transmission Electron Microscopy with a Diffraction-Grating Beam Splitter," Phys. Rev. Appl. 10, 061001 (2018).

4. B. J. McMorran, T. R. Harvey, C. Ophus, J. Pierce, and F. Yasin, "Demonstration of STEM Holography Using Diffraction Gratings," Microsc. Microanal. 24, 200-201 (2018).

5. A. Greenberg, F. Yasin, C. Johnson, and B. McMorran, "Lorentz Implementation of STEM Holography," Microsc. Microanal. 25, 96-97 (2019).

6. B. J. McMorran, "Seeing with Phase: Interferometric Electron Microscopy for Magnetic Materials and Biological Specimens," Microsc. Microanal. 25, 1210-1211 (2019).

7. S. A. Montoya, S. Couture, J. J. Chess, J. C. T. Lee, N. Kent, D. Henze, S. K. Sinha, M.-Y. Im, S. D. Kevan, P. Fischer, B. J. McMorran, V. Lomakin, S. Roy, and E. E. Fullerton, "Tailoring magnetic energies to form dipole skyrmions and skyrmion lattices," Phys. Rev. B 95, 024415 (2017).

8. J. Chess, S. Montoya, J. Lee, S. Roy, S. Kevan, E. Fullerton, and B. McMorran, "Observation of Skyrmions at Room-temperature in Amorphous Fe/Gd Films," Microsc. Microanal. 21, 1649-1650 (2015).

9. J. J. Chess, S. A. Montoya, T. R. Harvey, C. Ophus, S. Couture, V. Lomakin, E. E. Fullerton, and B. J. McMorran, "Streamlined approach to mapping the magnetic induction of skyrmionic materials," Ultramicroscopy 177, 78-83 (2017).

10. This work was supported by the National Science Foundation under Grant No. 1607733. 